\title{
Stabilization of Starobinsky-Vilenkin stochastic inflation by an environmental noise
}

\author{
Z. Haba \\ Institute of Theoretical Physics, University of Wroclaw, \\ 50-204 Wroclaw, Plac Maxa Borna 9, Poland \\ email:zhab@ift.uni.wroc.pl
}

February 13, 2019

\begin{abstract}
We discuss the inflaton $\phi$ in an interaction with an infinite number of fields treated as a random environment (noise) with a friction $\gamma^{2}>0$. In a Markovian approximation we obtain a stochastic wave equation (appearing also in the warm inflation models). After the replacement of the environment by the white noise the stochastic wave equation violates the energy conservation if $\gamma \neq 0$. We introduce a dark energy as a compensation of the inflaton energy-momentum. We add to the classical wave equation the Starobinsky-Vilenkin noise which in the slow-roll approximation describes the quantum fluctuations in an expanding metric. We investigate the resulting consistent stochastic Einstein-Klein-Gordon system in the slow-roll regime. We obtain Fokker-Planck equation for the probability distribution of the inflaton assuming that the dependence of the scale factor $a$ and the Hubble variable $H$ on the field $\phi$ is known. We obtain explicit stationary solutions of the Fokker-Planck equation assuming that $a(\phi)$ and $H(\phi)$ can approximately be determined in a slow roll regime with a neglect of noise. We extend the results to the multifield $D$-dimensional configuration space. We show that in the regime $a(\phi)^{3} H(\phi)^{5} \rightarrow \infty$ the quantum noise determines the asymptotic behaviour of the stationary distribution. If $a^{3} H^{5}$ stays finite then the environmental noise ensures the integrability of the stationary probability. In such a case there is no need to introduce boundary conditions with the purpose to eliminate infinite inflation. The variation of $a^{3} H^{5}$ could be interpreted as a sign of a transition from cold inflation to warm inflation.
\end{abstract}

\section{Introduction}

The inflationary model agrees very well with the observational data [1]. However, the model is a compilation of classical and quantum field theory. We 
consider an interaction of a classical scalar field with gravity in order to generate inflation and to derive the $\Lambda \mathrm{CDM}$ model. Then, quantization of scalar and tensor fields (linearized gravity) is introduced in order to explain the observed CMB fluctuations. These fluctuations agree with the observed CMB power spectrum (there are some difficulties with low multipoles). Starobinsky [2] [3] and Vilenkin [4] suggested an approach which treats quantum scalar field non-perturbatively in classical Einstein equations. Their idea is based on the observation that the quantum scalar field in an expanding universe behaves as a classical diffusion process. In such a case we obtain a stochastic EinsteinKlein-Gordon (EKG)system. In the slow-roll limit the stochastic EKG system is approximated by a diffusion equation.

In some approaches to cosmology it is pointed out that it is a paramount simplification to assume that there is only one field $\phi$ (inflaton) interacting with gravity. The inflaton as well as the observed luminous matter may be interacting with an infinite set of quantum fields creating particles which are not observed (this is consistent with the string theory point of view [5][6]). Each field gives a negligible contribution to the interaction but the infinite number of these fields forms an environment which can be treated by methods of statistical mechanics (central limit theorem) in a close analogy to the motion of a Brownian particle in a fluid [7][8]. As a result we obtain a stochastic wave equation for an inflaton with two perturbations: noise from the environment (thermal noise) and the quantum noise. The stochastic wave equation determines correlation functions which are measured in astronomical observations ,e.g., the power spectrum of fluctuations. In such a model one can ask questions intrinsic to a quantum theory, e.g., the probability of an appearance of the universe of a given size or the probability distribution of the inflaton [9]. The Starobinsky-Vilenkin theory gives an analytic formula for this probability at large time (stationary solution). The same result follows from quantization of gravity either on the basis of deWitt-Wheeler equation [10] or using the path-integral [11]. However, for some potentials the resulting stationary distribution is not normalizable. The result applies well in the range of slow-roll which coincides with the range of the field $\phi$ in the course of inflation. However, in order to restrict the range of evolution of $\phi$ in the stochastic equation we must introduce boundary conditions as is done in [12][13]. The theory of diffusions with boundary conditions is more involved [14] and no explicit estimates on the stationary probability distribution are available. We show in this paper that for some potentials which require boundary conditions in the approach [12]-[13] the environmental noise cures the integrability problem. In such a case the environmental noise could be treated as an alternative regularization procedure which gives some sense to the stationary evolution beyond the inflationary regime. We suggest the following interpretation: when the cold stochastic inflation of Starobinsky is terminating the warm inflation excited by the environmental noise dominates leading to a smooth behaviour at large time

In this paper we treat the stochastic wave equation with friction $\gamma>0$ and 
with thermal and quantum noise as an approximation to the Einstein-KleinGordon (EKG) equations. In such a case the energy-momentum tensor must be conserved. The noise in the inflaton equation violates the conservation law if $\gamma \neq 0$. We add to the energy-momentum of the inflaton $\phi$ a correction which ensures the conservation law and can be associated with the energy-momentum of the dark energy ( the idea expressed also in [15]). In such a case we can insert the stochastic field in Einstein equations arriving at stochastic Einstein equations. In a homogeneous flat universe we obtain a closed system of stochastic Friedmann equations. This system of equations determines the Fokker-Planck equation for the probability distribution. It contains many variables. In order to solve this equation we make some simplifying assumptions. The main approximation involves the assumption that the Hubble parameter $H(\phi)$ and the scale factor $a(\phi)$ can be expressed from the slow-roll no noise approximation of the EKG system. We show that the Fokker-Planck equation in a limit of small $a(\phi)^{3} H(\phi)^{5}$ has a stationary solution which has the same asymptotics as the solution we would have obtained solely from the thermal noise . For large $a^{3} H^{5}$ the asymptotics of the solution derived by Starobinsky (the HartleHawking probability density) results. The transition from large $a^{3} H^{5}$ to small $a^{3} H^{5}$ could be interpreted as a transition from cold inflation to warm inflation. The probability distribution of the inflaton gives a non-perturbative formula for quantum fluctuations with the thermal noise. Berera et al[16] [17] [18][19] calculate the power spectrum using the stochastic equation with the thermal noise (warm inflation). In [20] and [12] the power spectrum is calculated using the Starobinsky-Vilenkin approach to the cold inflation. There are some arguments [21] that the non-perturbative treatment of fluctuations can explain the low multipoles discord. From the calculations in [20][12] [18][19] [21] it can be seen that the addition of the thermal noise to cold inflation will change the power spectrum (we calculate it in a subsequent paper [22]).

We discuss a general framework of Einstein gravity with stochastic wave equations in secs.2-3. In sec.4 we make the slow-roll approximation for stochastic equations. We derive the Fokker-Planck equation assuming that the functions $a(\phi)$ and $H(\phi)$ are known. In sec.5 we obtain approximate formulas for these functions neglecting their noise dependence. We discuss the stationary solutions of the Fokker-Planck equation in sec.6.

\section{The energy-momentum tensor of the interac- tion with a random environment}

We consider an infinite set of scalar fields $\chi^{b}$ of mass $m_{b}$ coupling to the inflaton with an interaction Lagrangian $\mathcal{L}_{I}=\sum_{b} \lambda_{b} \chi^{b} \phi$ ( an outline of this scheme has been presented in [23], detailed calculations appeared in [24]). We solve the evolution equations for $\chi^{b}$ with the initial conditions whose probability distri- 
bution is defined by classical or quantum Gibbs distribution. In a Markovian approximation, assuming that $\gamma^{2} \simeq \sum_{b} \lambda_{b}^{2} m_{b}^{-2}<\infty$ and eliminating the fields $\chi^{b}$ from the equation of motion of the inflaton, we obtain in a flat expanding metric

$$
d s^{2}=g_{\mu \nu} d x^{\mu} d x^{\nu}=d t^{2}-a^{2} d \mathbf{x}^{2}
$$

a stochastic wave equation of the form (this equation has been derived earlier in $[25])$

$$
\partial_{t}^{2} \phi-a^{-2} \triangle \phi+\left(3 H+\gamma^{2}\right) \partial_{t} \phi+m^{2} \phi+V^{\prime}(\phi)+\frac{3}{2} \gamma^{2} H \phi=\eta
$$

where $H=a^{-1} \partial_{t} a$ and $\eta$ is a noise.

In an expanding universe the quantum field behaves like a classical diffusion process [3][4]. We can take into account quantum effects by an addition of the Starobinsky-Vilenkin noise to the rhs of eq.(1). Now,

$$
\eta \equiv \partial_{t} \xi=\gamma a^{-\frac{3}{2}} \partial_{t} B+\frac{3}{2 \pi} H^{\frac{5}{2}} \partial_{t} W
$$

where the first term describes the environmental noise and the second term the quantum noise. The factor $a^{-\frac{3}{2}}$ comes from det $\left|g_{\mu \nu}\right|^{\frac{1}{4}}$ and the factor $H^{\frac{5}{2}}$ is chosen in order to reproduce the correlation functions of the quantum scalar field in an expanding universe [26]. The (white) noise $\partial_{t} B$ is the the Gaussian random process with the covariance

$$
\left\langle\partial_{t} B \partial_{s} B\right\rangle=\delta(t-s)
$$

$\partial_{t} W$ is an independent Gaussian stochastic process with the same covariance $(3)$.

The energy-momentum tensor of the scalar field in the presence of friction $\gamma^{2} \partial_{t} \phi$ and noise $\xi$ is not conserved (as far as we know this point has not been discussed in papers on stochastic inflation). We have to compensate the energymomentum by means of a compensating energy-momentum $T_{d e}$ which we associate with the dark sector. Now, the conserved energy-momentum tensor $T_{\text {tot }}^{\mu \nu}$ is

$$
T_{t o t}^{\mu \nu}=T^{\mu \nu}+T_{d e}^{\mu \nu}
$$

where $T_{d e}$ denotes some other constituents of the primeval universe. From the conservation law

$$
\left(T_{d e}^{\mu \nu}\right)_{; \mu}=-\left(T^{\mu \nu}\right)_{; \mu}
$$

We assume the energy-momentum in the form of an ideal fluid

$$
T_{d e}^{\mu \nu}=\left(\rho_{d e}+p_{d e}\right) u^{\mu} u^{\nu}-g^{\mu \nu} p_{d e}
$$

where $\rho$ is the energy density and $p$ is the pressure. The velocity $u^{\mu}$ satisfies the normalization condition

$$
g_{\mu \nu} u^{\mu} u^{\nu}=1
$$


For the scalar field we have the representation (5) with

$$
\begin{gathered}
u^{\mu}=\partial^{\mu} \phi\left(\partial^{\sigma} \phi \partial_{\sigma} \phi\right)^{-\frac{1}{2}}, \\
\rho+p=\partial^{\sigma} \phi \partial_{\sigma} \phi, \\
p=\frac{1}{2} \partial^{\sigma} \phi \partial_{\sigma} \phi-V .
\end{gathered}
$$

If $\phi$ satisfies eq.(1) then we have for the scalar field (6)-(8)

$$
\left(T^{\mu \nu}\right)_{; \mu}=\partial^{\nu} \phi\left(\eta-\gamma^{2} \partial_{t} \phi-\frac{3}{2} \gamma^{2} H \phi\right) .
$$

We interpret eq.(9) as a stochastic equation in the Stratonowitch sense [14]. The Stratonowitch differential is the only one which satisfies the Leibniz rule. The divergence equation (9) for $T^{0 \nu}$ in a homogeneous metric in the frame $u=(1, \mathbf{0})$ (spatial homogeneity of $\phi$ ) gives (we apply the stochastic calculus and the ocircle notation for stochastic multiplication of ref. [14] )

$$
d \rho+3\left(1+w_{I}\right) H \rho d t=\partial_{t} \phi \circ d \xi-\frac{3}{2} \gamma^{2} H \phi \partial_{t} \phi d t-\gamma^{2}\left(\partial_{t} \phi\right)^{2} d t,
$$

For a scalar field with potential $V$ we have

$$
w_{I}=\left(\frac{1}{2}\left(\partial_{t} \phi\right)^{2}-V\right)\left(\frac{1}{2}\left(\partial_{t} \phi\right)^{2}+V\right)^{-1} .
$$

According to eq.(4) the compensating energy density must have the (non)conservation law with an opposite sign

$$
d \rho_{d e}+3 H(1+w) \rho_{d e} d t=\frac{3}{2} \gamma^{2} H \phi \partial_{t} \phi d t+\gamma^{2}\left(\partial_{t} \phi\right)^{2} d t-\partial_{t} \phi \circ d \xi
$$

where

$$
w=\frac{p_{d e}}{\rho_{d e}}
$$

is not determined by eq.(4). If a concrete model of the environmental scalar fields $\chi_{b}$ (discussed at the beginning of this section) is chosen then $w$ would be determined by this model. In order to have a closed system of equations an approximation $w \simeq$ const would be useful. For scalar fields $p+\rho=(1+w) \rho \simeq 0$ if the kinetic energy of the $\chi_{b}$ fields is negligible. We choose the interaction potential for the $\chi_{b}$ fields [24] which justifies the approximation $w \simeq-1$.

We have obtained in eqs.(10)-(12) a special form of the energy non-conservation. Some other models with a non-zero term on the rhs of eq.(10) (interpreted as a time derivative of the cosmological term) have been discussed [27][28][29][30]. In models of inflation [31][32][16] the term $\gamma^{2}\left(\partial_{t} \phi\right)^{2}$ describes a transformation of the inflaton energy into radiation. In such a case we add $\rho_{\text {rad }}$ to the total energy-momentum, omit the term $\gamma^{2}\left(\partial_{t} \phi\right)^{2}$ in the equation (12) for dark energy and in order to preserve the energy conservation we assume

$$
\partial_{t} \rho_{\text {rad }}+4 \rho_{\text {rad }}=\gamma^{2}\left(\partial_{t} \phi\right)^{2} .
$$


We insert the conserved energy-momentum in Einstein equations

$$
R^{\mu \nu}-\frac{1}{2} g^{\mu \nu} R=8 \pi G T_{t o t}^{\mu \nu},
$$

where $R^{\mu \nu}$ is the Ricci tensor and $G$ is the Newton constant. The Friedman equation in the FRLW flat metric reads

$$
H^{2}=\frac{8 \pi G}{3}\left(\rho+\rho_{d e}\right) .
$$

We differentiate eq.(15). Then, together with the stochastic equations (10) and (12) for the densities $\rho$, we have a closed system of stochastic equations

$$
\begin{gathered}
d a=H a d t \\
d H^{2}=\frac{8 \pi G}{3}\left(d \rho+d \rho_{d e}\right)=-8 \pi G H\left(\left(1+w_{I}\right) \rho+(1+w) \rho_{d e}\right) d t
\end{gathered}
$$

We can insert in eq.(17) the solution of eqs.(10),(12) for $\rho$ as a function of $a$ and subsequently insert $H$ as a function of $a$ obtaining a stochastic integrodifferential equation (16) for $a$. We choose our model of $\chi_{b}$ fields in such a way that $w \simeq-1$ in eqs.(12) and (17). Then, eq.(10) determines $\rho$ whereas eq.(17) does not depend explicitly on the dark energy. Eqs.(1),(7)-(8) and (16)-(17) (with $w=-1$ ) constitute a closed system of differential equations involving $\phi, H, a$ and the noise $\eta$.

\section{Stochastic Einstein-Klein-Gordon system}

Eq.(17) does not depend on the details of the compensating matter if $w=-1$. In such a case we obtain a closed random dynamical system (in the first order form) with the Starobinsky-Vilenkin noise $W$ and the environmental noise $B$

$$
\begin{gathered}
d \phi=\Pi d t, \\
d \Pi=-\left(3 H+\gamma^{2}\right) \Pi d t-V^{\prime} d t-\frac{3}{2} \gamma^{2} H \phi d t+\gamma a^{-\frac{3}{2}} \circ d B+\frac{3}{2 \pi} H^{\frac{5}{2}} \circ d W, \\
d H=-4 \pi G \Pi^{2} d t, \\
d a=H a d t .
\end{gathered}
$$

For a comparison with ref.[20] and a subsequent study of stochastic inflation it will be useful to change the world time $t$ into the e-folding time $\nu$ describing the change of the scale factor

$$
\nu=\int_{0}^{t} H d s=\ln \left(\frac{a}{a_{0}}\right) .
$$


In general relativity there is a freedom in the choice of the time variable. The e-folding time allows to integrate some stochastic equations [21][22]. This is also a proper choice of time for a description of cosmological perturbations and to calculate the power spectrum[20]. Then (for a simple description of a stochastic change of time see [33])

$$
\begin{gathered}
d \phi=H^{-1} \Pi d \nu, \\
d \Pi=-\left(3 H+\gamma^{2}\right) H^{-1} \Pi d \nu-V^{\prime} H^{-1} d \nu-\frac{3}{2} \gamma^{2} \phi d \nu \\
+\gamma \exp \left(-\frac{3}{2} \nu\right) H^{-\frac{1}{2}} \circ d B(\nu)+\frac{3}{2 \pi} H^{2} \circ d W(\nu), \\
d H=-4 \pi G \Pi^{2} H^{-1} d \nu .
\end{gathered}
$$

The stochastic wave equation leads to a definite relation between $\phi, \Pi, H$ and $a$. We multiply eq.(19) by $\Pi$ and using (20) we obtain the following identity (which could be considered as an equation for $H(\phi, \Pi, B, W)$ )

$$
\begin{aligned}
& \partial_{t}\left(\frac{1}{2} \Pi^{2}+V-\frac{3}{4} \gamma^{2} H \phi^{2}-\frac{3}{8 \pi G} H^{2}+\frac{1}{4 \pi G} \gamma^{2} H\right)-3 \pi G \gamma^{2} \phi^{2} \Pi^{2} \\
& =\gamma a^{-\frac{3}{2}} \Pi \circ \partial_{t} B+\frac{3}{2 \pi} H^{\frac{5}{2}} \Pi \circ \partial_{t} W .
\end{aligned}
$$

From eqs.(24)-(25) we obtain a similar equation for the $\nu$-derivative

$$
\begin{aligned}
& \partial_{\nu}\left(\frac{1}{2} \Pi^{2}+V-\frac{3}{4} \gamma^{2} H \phi^{2}-\frac{3}{8 \pi G} H^{2}+\frac{1}{4 \pi G} \gamma^{2} H\right)-3 \pi G \gamma^{2} H^{-1} \phi^{2} \Pi^{2} \\
& =\gamma \exp \left(-\frac{3}{2} \nu\right) H^{-\frac{1}{2}} \Pi \circ \partial_{\nu} B+\frac{3}{2 \pi} H^{2} \Pi \circ \partial_{\nu} W
\end{aligned}
$$

Integrating eq.(27)

$$
\begin{aligned}
& \frac{1}{2} \Pi^{2}+V-\frac{3}{4} \gamma^{2} H \phi^{2}-\frac{3}{8 \pi G} H^{2}+\frac{1}{4 \pi G} \gamma^{2} H+V_{0} \\
& =3 \pi G \gamma^{2} \int_{0}^{\nu^{4}} d s H^{-1} \phi^{2} \Pi^{2}+\int_{0}^{\nu} d s\left(\gamma \exp \left(-\frac{3}{2} s\right) H^{-1} \Pi \circ d B+\frac{3}{2 \pi} H^{2} \Pi \circ d W\right),
\end{aligned}
$$

(where $V_{0}$ is an arbitrary integration constant related to the cosmological constant $\Lambda$ by $8 \pi G V_{0}=\Lambda$ ). Eqs.(26)-(27) can be considered as equations for $H$. We can get a solution of eq.(27) as a perturbation series in $\gamma$. At $\gamma=0$ eq.(27) has the solution

$$
\begin{aligned}
& \left(\frac{3}{8 \pi G} H^{2}-\frac{1}{2} \Pi^{2}-V-V_{0}\right)(\nu) \\
& =\left(\frac{3}{8 \pi G} H^{2}-\frac{1}{2} \Pi^{2}-V-V_{0}\right)(0) \exp \left(-4 G \int_{0}^{\nu} \Pi(s) \circ d W(s)\right) \\
& -4 G \int_{0}^{\nu}\left(\frac{1}{2} \Pi^{2}+V+V_{0}\right) \exp \left(-4 G \int_{s}^{\nu} \Pi(\tau) \circ d W(\tau)\right) \Pi(s) \circ d W(s),
\end{aligned}
$$

where the first term on the rhs corresponds to the initial condition for the lhs. Inserting the expansion $H_{\gamma}=H+\gamma H_{1}+\ldots$ in eq.(28) we obtain $H(\phi, \Pi, W, B)$ as a power series in $\gamma$. Then, eqs.(23)-(24) define a non-linear stochastic wave equation for $(\phi, \Pi)$. The solution allows to calculate the probability distribution of $(\phi, \Pi)$. 


\section{Stochastic equations for slow-roll inflation}

The program outlined at the end of sec.3 to solve the stochastic Einstein-KleinGordon (EKG) equation and calculate the probability distribution is difficult to perform in a non-perturbative way. We shall rely on approximations. First, we neglect the noise in eqs.(27)-(28) and the term $\gamma^{2} \Pi^{2} \phi^{2}$. Then,

$$
H=\gamma^{2}\left(\frac{1}{3}-4 \pi G \phi^{2}\right)+\sqrt{\frac{8 \pi G}{3}\left(V+V_{0}\right)+\frac{1}{2} \Pi^{2}+\gamma^{4}\left(\frac{1}{3}-4 \pi G \phi^{2}\right)^{2}} .
$$

Using, eq.(30) we can obtain the Fokker-Planck equation for the probability distribution of $(\phi, \Pi)$ but it would be difficult to find solutions of this equation. We make the next simplifying assumption $\Pi \simeq 0$ and $\gamma=0$ in eq.(30). Then, the stochastic system (19)-(21) is

$$
\begin{gathered}
3 H d \phi=-V^{\prime} d t+\gamma a^{-\frac{3}{2}} \circ d B+\frac{3}{2 \pi} H^{\frac{5}{2}} \circ d W \\
d H=-4 \pi G\left(\partial_{t} \phi\right)^{2} d t \\
d a=H a d t .
\end{gathered}
$$

In the e-fold time the $\Pi \simeq 0$ limit reads (we express $a$ as a function of $\phi$ )

$$
\begin{gathered}
3 H d \phi=-V^{\prime} H^{-1} d \nu+\gamma a^{-\frac{3}{2}} H^{-\frac{1}{2}} \circ d B(\nu)+\frac{3}{2 \pi} H^{2} \circ d W(\nu), \\
d \ln (H)=-4 \pi G\left(\partial_{\nu} \phi\right)^{2} d \nu .
\end{gathered}
$$

The approximation $\Pi \simeq 0$ together with

$$
H=\sqrt{\frac{8 \pi G}{3}\left(V+V_{0}\right)}
$$

is known as the slow-roll approximation. Note that eq.(36) follows from eq.(29) and the assumption $\Pi \simeq 0$. The slow-roll approximation fails if the variables $\epsilon=$ $\frac{1}{16 \pi G}\left(V^{\prime}\right)^{2}\left(V+V_{0}\right)^{-2}$ and $\tilde{\eta}=\frac{1}{8 \pi G} V^{\prime \prime}\left(V+V_{0}\right)^{-1}$ are of order 1 . For deterministic systems we can restrict the initial values of the field and the time evolution in order to satisfy the requirement of small $\epsilon$ and $\tilde{\eta}$. It is more difficult to do it in a random system because the noise can move the system to the forbidden region of the field configurations. We can define the stochastic system in a required domain of field configurations by an imposition of boundary conditions (as discussed in [12][13] for the Starobinsky-Vilenkin stochastic equation with $\gamma=0$ ). However, the stochastic process and its probability distribution depend on the boundary conditions, e.g., for some potentials $V$ the limit $t \rightarrow \infty$ (the stationary distribution) exists for the process with the boundary conditions but does not exist if the boundary conditions are removed. Nevertheless, some correlation functions may have a negligible dependence on boundary conditions 
as discussed in [12][13] (for more on boundaries in diffusions see [14]). We show in the next section that in some models of inflation the assumption of $\gamma \neq 0$ allows to work without boundary conditions.

The formula (36) for $H$ has been applied by most authors on stochastic inflation. It can be considered as a definition of $V$ in the Hamiltonian-Jacobi framework [34][35][36]. It can be shown that eq.(36) is exact in a deterministic EKG system at large time [37]. If $H$ is known then in principle we can determine $a(\phi)$ (from eq.(33)) after solving the stochastic equations. We can obtain an explicit formula if we neglect the noise (as in eq.(36)) and apply the slow roll approximation (31) (without noise). Then,

$$
\ln (a)=\int H d t=\int d \phi\left(\frac{d \phi}{d t}\right)^{-1} H=-8 \pi G \int d \phi\left(V+V_{0}\right)\left(V^{\prime}\right)^{-1} .
$$

As discussed in sec.3 the correction to eq.(37) could be calculated as a perturbation series in the noise. Eq.(37) can be derived also from the exact formula for the mean value of the e-folds as the leading term of the saddle point expansion $[20][22]$.

We can generalize the stochastic equations (31) to multiple scalar fields $[38][13] \phi=\left(\phi^{1}, \ldots, \phi^{D}\right)$. Then, the noise $\eta=\left(\eta^{1}, \ldots, \eta^{D}\right)$ (eq. $\left.(2)\right)$ consists of independent random Gaussian variables with the same variance. In eq.(31) $\phi$ and the noises should be treated as vectors, $V^{\prime} \rightarrow \nabla V$ and $\left(\partial_{t} \phi\right)^{2} \rightarrow\left|\partial_{t} \phi\right|^{2}$ (the length of the vector $\left.\partial_{t} \phi\right)$. We can calculate $a(\phi)$ if $V=V(|\phi|)$ is rotation invariant with

$$
|\phi|^{2}=\left(\phi^{1}\right)^{2}+\ldots+\left(\phi^{D}\right)^{2}
$$

Then, (after an omission of noise in eq.(31))

$$
\ln (a)=\int H d t=\int d|\phi|\left(\frac{d|\phi|}{d t}\right)^{-1} H=-8 \pi G \int d|\phi|\left(V+V_{0}\right)\left(\frac{d V}{d|\phi|}\right)^{-1}
$$

For the system (31)-(33) we have the Fokker-Planck equation [39] for the probability distribution of $\phi$ ( we assume the Stratonovich interpretation of the stochastic equations [14])

$$
\partial_{t} P=\frac{\gamma^{2}}{18} \partial_{\phi} \frac{1}{H a^{\frac{3}{2}}} \partial_{\phi} \frac{1}{H a^{\frac{3}{2}}} P+\frac{1}{8 \pi^{2}} \partial_{\phi} H^{\frac{3}{2}} \partial_{\phi} H^{\frac{3}{2}} P+\partial_{\phi}(3 H)^{-1} V^{\prime} P .
$$

In the e-folding time eq.(34) determines the probability distribution as a solution of the equation

$$
\partial_{\nu} P=\frac{\gamma^{2}}{18} \partial_{\phi} \frac{1}{H^{\frac{3}{2}} a^{\frac{3}{2}}} \partial_{\phi} \frac{1}{H^{\frac{3}{2}} a^{\frac{3}{2}}} P+\frac{1}{8 \pi^{2}} \partial_{\phi} H \partial_{\phi} H P+\partial_{\phi}\left(3 H^{2}\right)^{-1} V^{\prime} P
$$

In the multifield case $\left(\phi \in R^{D}\right)$ with $\partial_{j}=\frac{\partial}{\partial \phi^{j}}$ eq.(39) reads

$$
\partial_{t} P=\sum_{j} \partial_{j}\left(\frac{\gamma^{2}}{18} \frac{1}{H a^{\frac{3}{2}}} \partial_{j} \frac{1}{H a^{\frac{3}{2}}} P+\frac{1}{8 \pi^{2}} H^{\frac{3}{2}} \partial_{j} H^{\frac{3}{2}} P+(3 H)^{-1} \partial_{j} V\right) P .
$$




\section{The evolution of the scale factor $a(\phi)$ in some inflationary models}

We express $H(\phi)$ as a function of $\phi$ from eq.(36). The dependence of $a$ on $\phi$ is more involved. We determine it from eq.(37) obtained in the slow-roll approximation and no noise limit. All the formulas in this section can be generalized to a multifield case (38) just by a replacement $\phi \rightarrow|\phi|$. Let us consider some examples of potentials appearing in inflation models [6][36][40][41][42][43]. If $V=\frac{m^{2}}{2} \phi^{2}$ ( a chaotic inflation [44] ) then from eq.(37) (the cosmological constant $\left.\Lambda=8 \pi G V_{0}\right)$

$$
a=\exp \left(-4 \pi G V_{0} m^{-2} \ln \phi^{2}-2 \pi G \phi^{2}\right) .
$$

Large $\phi$ corresponds to small $a$ and small $\phi$ to large $a$. If $V=g \phi^{n}(n>2)$ then

$$
a=\exp \left(-\frac{8 \pi G V_{0}}{(2-n) n g} \phi^{2-n}-4 \pi G n^{-1} \phi^{2}\right) .
$$

If $\phi \rightarrow \infty$ then $a \rightarrow 0$, if $\phi \rightarrow 0$ then $a \rightarrow \infty$ (for $\Lambda>0$ ).

If $V=g \exp (\lambda \phi)$ then

$$
a=\exp \left(\frac{8 \pi G V_{0}}{g \lambda^{2}} \exp (-\lambda \phi)-\frac{8 \pi G}{\lambda} \phi\right) .
$$

If $\phi \rightarrow+\infty$ then $a \rightarrow 0$, if $\phi \rightarrow-\infty$ then $a \rightarrow \infty$.

For a flat (plateau) potential [6][40]

$$
V=\frac{L+\phi^{2}}{K+\phi^{2}}
$$

we have

$$
\begin{aligned}
& a=\exp \left(-8 \pi G\left(\frac{V_{0} K^{2}}{4(K-L)} \ln \phi^{2}+\frac{V_{0} K}{2(K-L)} \phi^{2}+\frac{V_{0}}{8(K-L)} \phi^{4}+\frac{K L}{4(K-L)} \ln \phi^{2}\right.\right. \\
& \left.\left.+\frac{K+L}{4(K-L)} \phi^{2}+\frac{1}{8(K-L)} \phi^{4}\right)\right) .
\end{aligned}
$$

If $K>L \geq 0$ then $a \rightarrow 0$ if $\phi \rightarrow \infty$ and $a \rightarrow \infty$ if $\phi \rightarrow 0$. If $L>K>0$ then $a \rightarrow \infty$ if $\phi \rightarrow \infty$ and $a \rightarrow 0$ if $\phi \rightarrow 0$. If $0<K<L$ then $V^{\prime}<0$ and this is the reason why $a$ is increasing as a function of $\phi$.

Let $V=g \cos \phi$ then

$$
a=\exp \left(-8 \pi G\left(-g^{-1} V_{0} \ln \left|\tan \left(\frac{\phi}{2}\right)\right|-\ln |\sin (\phi)|\right)\right) .
$$

$a \rightarrow 0$ when $\phi \rightarrow 0$. When $\phi \rightarrow \pi$ then $a$ may go to $\infty$ if $g^{-1} \Lambda$ is large enough (otherwise $a \rightarrow 0$ ). 
The special case

$$
V=|g|(1-\cos \phi)
$$

corresponds to the "natural inflation" [45] describing the axion inflation . From eq.(37) we obtain

$$
a=\exp \left(8 \pi G \ln \left(2 \cos ^{2}\left(\frac{\phi}{2}\right)\right)\right) .
$$

Here, $-\pi \leq \phi \leq \pi$. From the classical dynamics $|\phi|$ is decreasing in time and $|\phi| \rightarrow 0$ for large time. So, $\phi$ tends to the minimum of the potential achieving a maximal (finite) value of $a$ at the minimum of the potential.

For the double well potential

$$
\begin{gathered}
V(\phi)=\frac{g}{4} \phi^{4}-\frac{\mu^{2}}{2} \phi^{2}=\frac{g}{4}\left(\phi^{2}-\frac{\mu^{2}}{g}\right)^{2}-\frac{\mu^{4}}{4 g} . \\
a=|\phi|^{\frac{8 \pi G V_{0}}{\mu^{2}}}\left|g \phi^{2}-\mu^{2}\right|^{\frac{\pi G \mu^{2}}{g}-\frac{4 \pi G V_{0}}{\mu^{2}}} \exp \left(-\pi G \phi^{2}\right) .
\end{gathered}
$$

If $\phi \rightarrow 0$ then $a \rightarrow 0$ (for $V_{0}>0$, if $V_{0}=0$ then $a \rightarrow$ const $\neq 0$ ). If $\phi \rightarrow \mu g^{-\frac{1}{2}}$ then $a$ goes either to 0 or to infinity depending on the value of $V_{0}$ (for $V_{0}=\frac{\mu^{4}}{4 g}$ we have $a \rightarrow$ const $\neq 0)$. When $\phi \rightarrow \infty$ then $a \rightarrow 0$. According to the formula (31) $(B=W=0)$ the classical slow roll time evolution of $\phi$ is determined by

$$
-\sqrt{24 \pi G} \frac{d \phi}{d t}=\phi\left(g \phi^{2}-\mu^{2}\right)\left(V_{0}+\frac{g}{4} \phi^{4}-\frac{\mu^{2}}{2} \phi^{2}\right)^{-\frac{1}{2}}
$$

Hence, if $\mu g^{-\frac{1}{2}} \geq \phi \geq 0$ then $\phi$ is increasing. If $\phi \geq \mu g^{-\frac{1}{2}}$ then $\phi$ is decreasing to $\mu g^{-\frac{1}{2}}$. It follows that $\phi(t) \rightarrow \mu g^{-\frac{1}{2}}$ when $t \rightarrow \infty$. If $V_{0}=\frac{\mu^{4}}{4 g}$ then $V+V_{0} \rightarrow 0$ for $t \rightarrow \infty$. Then, the slow-roll condition is violated for a large time (this remains true for $\mu=0$ ). Nevertheless, the stochastic dynamical system (31) still makes sense. As will be shown in the next section the stochastic equation with the Starobinsky-Vilenkin noise leads to a non-integrable stationary probability distribution (if treated as a system on the whole real line) whereas the system with the environmental noise has a normalizable stationary distribution.

\section{Stationary probability distribution of the in- flaton}

The probability distribution $P_{t}\left(\phi, \phi_{0}\right)$ determines the probability of an appearance of the universe with given $\phi$ ( or $a(\phi))$ when initially it had the value $\phi_{0}$. The stationary probability $P(\phi)$ is the limit of $P_{t}$ for $t \rightarrow \infty$ [39] (it does not depend on $\left.\phi_{0}\right)$. In the cosmic time and with the Stratonovitch interpretation it can be obtained from the requirement $\partial_{t} P=0$ which gives (after an integration over $\phi$ )

$$
\frac{\gamma^{2}}{18} \frac{1}{H a^{\frac{3}{2}}} \partial_{\phi} \frac{1}{H a^{\frac{3}{2}}} P+\frac{1}{8 \pi^{2}} H^{\frac{3}{2}} \partial_{\phi} H^{\frac{3}{2}} P+(3 H)^{-1} V^{\prime} P=0
$$


For the e-fold time

$$
\frac{\gamma^{2}}{18} \frac{1}{H^{\frac{3}{2}} a^{\frac{3}{2}}} \partial_{\phi} \frac{1}{H^{\frac{3}{2}} a^{\frac{3}{2}}} P+\frac{1}{8 \pi^{2}} H \partial_{\phi} H P+(3 H)^{-2} V^{\prime} P=0 .
$$

In the multidimensional case (41) the requirement $\partial_{t} P=0$ is satisfied if

$$
\frac{\gamma^{2}}{18} \frac{1}{H a^{\frac{3}{2}}} \partial_{j} \frac{1}{H a^{\frac{3}{2}}} P+\frac{1}{8 \pi^{2}} H^{\frac{3}{2}} \partial_{j} H^{\frac{3}{2}} P+(3 H)^{-1} \partial_{j} V P=0 .
$$

This is a general form of the equation for rotationally invariant stationary probability. If the functions $P$ and $V$ depend only on $|\phi|$ then eq.(53) is equivalent to

$$
\frac{\gamma^{2}}{18} \frac{1}{H a^{\frac{3}{2}}} \partial_{|\phi|} \frac{1}{H a^{\frac{3}{2}}} P+\frac{1}{8 \pi^{2}} H^{\frac{3}{2}} \partial_{|\phi|} H^{\frac{3}{2}} P+(3 H)^{-1} P \partial_{|\phi|} V=0 .
$$

In our model of diffusion let us consider the simplest cases first. The stationary solution of eq.(51) without the Starobinsky-Vilenkin noise is

$$
\begin{aligned}
& P=\sqrt{V+V_{0}} \exp \left(-12 \pi G \int^{\phi} d \phi^{\prime}\left(V^{\prime}\right)^{-1}\left(V+V_{0}\right)\right) \\
& \exp \left(-\frac{6}{\gamma^{2}} \sqrt{\frac{8 \pi G}{3}} \int d \phi V^{\prime} \sqrt{V+V_{0}} \exp \left(-24 \pi G \int^{\phi} d \phi^{\prime}\left(V^{\prime}\right)^{-1}\left(V+V_{0}\right)\right),\right.
\end{aligned}
$$

where the exponential factors in eq.(55) come from the formula for $a^{3}$ (eq.(37)). If we assume that $V$ does not grow faster than exponentially and is an even function of $\phi$ then for a large $|\phi|$

$$
P \simeq \sqrt{V+V_{0}} \exp \left(-12 \pi G \int^{\phi} d \phi^{\prime}\left(V^{\prime}\right)^{-1}\left(V+V_{0}\right)\right)
$$

because the last factor in eq.(55) tends to 1 . If $\gamma=0$ (the environmental noise is absent) then we obtain the Starobinsky solution (discussed also by Vilenkin [4] and Linde [9]) which in the Stratonovitch interpretation takes the form

$$
P=\left(V+V_{0}\right)^{-\frac{3}{4}} \exp \left(\frac{3}{8 G^{2}} \frac{1}{V+V_{0}}\right) .
$$

Ito interpretation gives the factor $\left(V+V_{0}\right)^{-\frac{3}{2}}$ whereas e-fold time the factor $\left(V+V_{0}\right)^{-1}$ in front of the exponential. The probability distribution (57) is also the stationary solution in the multifield rotationally symmetric D-dimensional case (54). Then, for $V \simeq|\phi|^{n}$ it fails to be integrable at large $|\phi|$ if $\frac{3}{4} n \leq D$ in Stratonovitch case and $n \leq D$ in the e-fold time (as discussed in [12][13]). The authors [12][13] impose boundary conditions at large $\phi$ and discuss whether the dependence on boundary conditions has consequences on some measurable expectation values. In the system with an environmental noise and the $\phi^{n}$ potentials we stabilize the system by the environmental noise instead of restricting it by boundary conditions (when $P$ in eq.(57) is not integrable). The environmental noise is present in all physical systems. Its crucial role in equilibration of dynamical systems is well-known [46]. Its action can really be seen as a stabilization because some correlation functions will have a singular or chaotic behaviour 
at $t \rightarrow \infty$ when $\gamma=0$ and a smooth behaviour when $\gamma>0$ (the same remark applies to boundary conditions;some correlation functions with boundary conditions are discussed in [13] ). If $D=1$ and $\left(V+V_{0}\right)=\phi^{n}$ with $n \geq 2$ then $P$ is integrable at large $\phi$ but not integrable at $\phi=0$. $\phi \simeq 0$ does not satisfy the slow-roll conditions. We could remove it from the configuration space imposing boundary conditions at $\phi \simeq 8 \pi G$. However, such boundary conditions change the stochastic equations [14] and the equations for probability distributions. In general, it is not simple to estimate the stationary probability distribution. The formula (57) fails to express a probability distribution ( $P$ is not integrable) if $V$ does not fall quickly enough for large $\phi$. This is the case with the exponential potential (discussed at eq.(44)) when $\phi \rightarrow-\infty$ and with the flat potential (45). Moreover, $P$ in eq.(57) may be non-integrable at finite $\phi$ if $V+V_{0}=0$ at a certain $\phi_{c}$ as for the natural inflation (47) and the double well potential (48). The non-integrability occurs in the range of $\phi$ which is outside of the slow-roll regime determined by the small values of the parameters $\epsilon$ and $\tilde{\eta}$. We could expect that $P$ of eq.(57) is still an approximate stationary probability (after an imposition of boundary conditions) in the slow-roll region and integrable there. We shall show that for some potentials the environmental noise leads to explicit formulas for the stationary probability without the problems with integrability. For this reason the introduction of the environmental noise could be considered as an alternative method of stabilization of the probability distribution. It allows an extension of the stochastic equation beyond the slow-roll (or inflation) region.

With $\gamma \neq 0$ for the Stratonovitch equation (51) we write

$$
\tilde{P}=H^{-1} a^{-\frac{3}{2}} P \text {. }
$$

Then, the equation for $\tilde{P}$ is

$$
\frac{\gamma^{2}}{18} H^{-1} a^{-\frac{3}{2}} \partial_{\phi} \tilde{P}+\frac{1}{8 \pi^{2}} H^{\frac{3}{2}} \partial_{\phi}\left(H^{\frac{5}{2}} a^{\frac{3}{2}} \tilde{P}\right)=-\frac{1}{3} V^{\prime} a^{\frac{3}{2}} \tilde{P} .
$$

Using the formulas for $H$ and for $a$ (eq.(37)) we obtain

$$
\begin{aligned}
& \ln \tilde{P}=-6 \int d \phi H a^{3}\left(\gamma^{2}+\frac{9}{4 \pi^{2}} H^{5} a^{3}\right)^{-1} \\
& \left(V^{\prime}+\frac{10}{3} G^{2}\left(V+V_{0}\right) V^{\prime}-32 \pi G^{3}\left(V+V_{0}\right)^{3}\left(V^{\prime}\right)^{-1}\right) .
\end{aligned}
$$

For most potentials $a \rightarrow 0$ for $\phi \rightarrow \infty$ then $a^{3} H^{5} \rightarrow 0$ and we get from eq.(59) $P \simeq H a^{\frac{3}{2}}$ coinciding with the formula (56). It leads to stationary solutions which are integrable at large $\phi$. As an explicit example we consider the chaotic inflation potential $\phi^{n}$ with $V_{0}=0$. Then, the formula for $a(43)$ gives for a large $\phi($ small $a)$

$$
P=|\phi|^{\frac{n}{2}} \exp \left(-6 \pi G n^{-1} \phi^{2}\right) .
$$

At small $\phi$ and $V_{0}=0$ we have $a^{3} H^{5} \rightarrow 0$. So, the formulae (56) and (60) are applicable for small as well as large $\phi$.The formula (60) (which holds true for 
multidimensional fields $\phi$ ) describes in statistics the gamma distribution ( more precisely the $\chi^{2}$ distribution). The Starobinsky formula (57) (obtained without the thermal noise) gives $P$ which is not integrable at $\phi=0$. If $V_{0} \neq 0$ for $\phi^{n}$ potential then $a(\phi) \rightarrow \infty$ for $\phi \rightarrow 0$ and $a^{3} H^{5} \rightarrow \infty$. Then, the formula (57) applies, but with $V_{0}>0$, hence it is integrable.

In general, when $a^{3} H^{5} \rightarrow \infty$ then

$$
\ln \tilde{P} \simeq-\frac{8 \pi^{2}}{3} \int d \phi H^{-4}\left(V^{\prime}+\frac{10}{3} G^{2}\left(V+V_{0}\right) V^{\prime}-32 \pi G^{3}\left(V+V_{0}\right)^{3}\left(V^{\prime}\right)^{-1}\right)
$$

We can calculate the rhs of eq.(61) and convince ourselves that in this case we reach the Starobinsky formula (57).

We could set

$$
\hat{P}=H^{\frac{3}{2}} P
$$

then the equation for $\hat{P}$ reads

$$
\frac{\gamma^{2}}{18} H^{-1} a^{-\frac{3}{2}} \partial_{\phi}\left(H^{-\frac{5}{2}} a^{-\frac{3}{2}} \hat{P}\right)+\frac{1}{8 \pi^{2}} H^{\frac{3}{2}} \partial_{\phi} \hat{P}=-\frac{1}{3} H^{-\frac{5}{2}} V^{\prime} \hat{P}
$$

When we calculate the derivatives of $H$ and $a$ then we obtain

$$
\begin{aligned}
& \left(\gamma^{2}+\frac{9}{4 \pi^{2}} a^{3} H^{5}\right) \partial_{\phi} \ln \hat{P} \\
& =-6 H a^{3}\left(V^{\prime}+2 \pi G \gamma^{2} a^{-3} H^{-1}\left(V+V_{0}\right)\left(V^{\prime}\right)^{-1}-\gamma^{2} \frac{5}{24} H^{-1}\left(V+V_{0}\right)^{-1} V^{\prime}\right)
\end{aligned}
$$

From eq.(62) we can also see that if $a \rightarrow 0$ for a large $\phi$ then

$$
P \simeq H a^{\frac{3}{2}}
$$

in agreement with eq.(56).

In the e-fold equation (52) we write

$$
P=H^{\frac{3}{2}} a^{\frac{3}{2}} P_{e}
$$

Then, the equation for $P_{e}$ reads

$$
\begin{aligned}
& -\partial_{\phi} \ln P_{e}=-6\left(\gamma^{2}+\frac{9}{4 \pi^{2}} a^{3} H^{5}\right)^{-1} \\
& H a^{3}\left(V^{\prime}+\frac{10}{3} G^{2}\left(V+V_{0}\right) V^{\prime}-32 \pi G^{3}\left(V+V_{0}\right)^{3}\left(V^{\prime}\right)^{-1}\right)
\end{aligned}
$$

There remains to explore the formula (62) for large $a$ in various models. If $a^{3} V^{\frac{5}{2}} \rightarrow \infty$ then the terms independent of $a$ in eq.(62) can be omitted and we get the Starobinsky formula (57) as we did on the basis of eq.(61). As expected eqs.(59) and (62) lead to the same formula for $P$ but they may be applied for direct estimates in different asymptotic regions. 
Let us still investigate the stationary probability distributions for the remaining potentials of sec.5. For the flat potential (45) the Starobinsky formula (57) is not integrable at large $\phi$. However, if $K>L \geq 0$ then $a(\phi) \rightarrow 0$ and $a^{3} H^{5} \rightarrow 0$. Hence, the distribution (56) which is integrable applies at large $\phi$. However, if $L>K>0$ then $a^{3} H^{5} \rightarrow \infty$. Then, for a large $\phi$ the Starobinsky formula (57) arises as a limit which is not integrable. This example shows that the thermal noise stabilization is not universal (it seems to apply if $a$ does not grow with $\phi$ ). In the exponential model $a \rightarrow 0$ if $\phi \rightarrow+\infty$. Then, $a^{3} H^{5} \rightarrow 0$ and eq. (56) applies. Hence, $P$ is integrable at large positive $\phi . a(\phi)$ tends to infinity if $\phi \rightarrow-\infty$. If $V_{0}>0$ then $a^{3} H^{5} \rightarrow \infty$. The asymptotic behaviour of $P$ is determined by the distribution (57) which is not integrable at large negative $\phi$. In the double well model if $V_{0}=\frac{\mu^{4}}{4 g}$ then the Starobinsky distribution (57) would not be integrable because of the singularity at $V+V_{0} \simeq 0$. However, $a^{3} H^{5}$ does not tend to infinity. Hence, eq.(62) applies which leads to an integrable stationary distribution (approximated by eq.(56)). The Starobinsky solution (57) for the natural inflation (47) behaves for a small $\phi$ as

$$
P \simeq|\phi|^{-\frac{3}{2}} \exp \left(\frac{16}{3 G^{2} \phi^{2}}\right)
$$

Hence, it is not integrable. The environmental noise changes the small $\phi$ behaviour (as follows from eqs. (59) and(62)) (because $a^{3} V^{\frac{5}{2}}$ is negligible in comparison to $\gamma^{2}$ for small $\phi$ ). From eq.(62) for a small $\phi$

$$
P \simeq|\phi|
$$

Hence, $P$ as determined by eq. (62) is a well-defined probability measure on the interval $-\pi \geq \phi \leq \pi$.

Summarizing, if $a^{3} H^{5}$ tends to zero we obtain the formula (56) (either for small or large $\phi$ ) with an integrable $P$. If $a^{3} H^{5}$ tends to infinity then asymptotically the behaviour of $P$ is determined by the Starobinsky formula (57) which may fail the integrability condition. In the last case the boundary conditions would be necessary in order to obtain an integrable stationary distribution.

\section{Summary and conclusions}

The Starobinsky-Vilenkin stochastic equation is a useful tool in a study of an expanding universe at an early quantum stage. Recently, it has been applied to the calculation of correlation functions of e-folds and the power spectrum [20][12][13][47][48]. For some potentials the stationary probability distribution is not integrable when applied to the whole range of field values. For a single inflaton field the non-integrability occurs for polynomial large field values only if $n<2$ (e-fold time). For small field values the non-integrability is beyond the 
range of the slow-roll regime when the inflation stops. In the case of multidimensional inflaton field the non-integrability appears at large field values when the standard slow roll conditions are satisfied [12][13]. A way out of this situation (as suggested in [12][13]) is to apply the stochastic equations in a region with boundaries excluding the forbidden regions. However, such a procedure modifies the stochastic equations and does not lead to explicit estimates on the stationary probability distribution. Our work suggests another approach which to some extent is implicit also in the warm inflation. When the cold inflation stops the environmental (thermal) noise becomes relevant. In [12] the appearance of non-integrability (infinite inflation) is compared to a phase transition in solid state physics. It is known [46] that close to the critical point the environment in the form of noise or boundaries (as, e.g., in the Ising model) becomes relevant. This is exactly what happens in the models discussed in this paper. When the field value goes beyond the inflation range the boundary conditions become relevant (in the framework of [12][13]) or in our alternative formulation the environmental noise begins to play its role. The stationary probability depends substantially on the noise although correlation functions of some observables may only marginally depend of it. We think that the environmental noise present in any (not idealized) physical system is a better tool than some arbitrary boundary conditions. The thermal noise accompanies classical as well as quantum field theories when an interaction with an environment is taken into account. The environment may consist of any degrees of freedom which undergo an averaging procedure. We have shown that the addition of the thermal noise has a stabilizing effect on quantum fluctuations. In sec.4 we have shown that if $a^{3} H^{5}$ stays bounded then the thermal noise repairs $P$ even beyond the slow-roll regime.The non-integrability of the stationary solution excludes it from calculation of expectation values. In such a case we cannot expect a finite result for the power spectrum at $t \rightarrow \infty$ (it is calculated in [20][48] at finite time). The standard estimates of the slow roll parameters $\epsilon \simeq\left(V^{-1} V^{\prime}\right)^{2}$ and $\tilde{\eta} \simeq V^{-1} V^{\prime \prime}$ restrict the values of fields in the form of boundaries but they could be replaced by expectation values which could be small because the forbidden values of the fields would have small probabilities with the presence of the thermal noise.

\section{References}

[1] P.A.R. Ade et al, arXiv:1502.01589

[2] A.A. Starobinsky, Phys.Lett.B117,175(1982)

[3] A.A.Starobinsky, in Current Topics in Field Theory,Quantum Gravity and Strings, ed. By H.J.Vega and N. Sanchez, Lecture Notes in Phys.246, Springer, 1986

[4] A. Vilenkin, Phys.Rev. D27,2848(1983) 
[5] D. Mitchell and N. Turok, Phys.Rev.Lett.58,1577(1987)

A. Berera and T.W. Kephart, Phys.Rev.Lett.83,1084(1999)

[6] E. Silverstein, TASI Lectures, arXiv:1606.0364

[7] G.W. Ford and M. Kac, Journ.Stat.Phys.46,803(1987)

[8] G.W. Ford, J.T. Lewis and R.F. O'Connell,

[9] A. D. Linde, Phys.Rev. D58,083514(1998)

A. D. Linde, Phys.Rev. D49,748(1994)

[10] S. Weinberg, Rev.Mod.Phys.61,1(1989)

[11] J.B.Hartle and S.W. Hawking, Phys.Rev.D28,2960(1983) Phys.Rev. A37,4416(1988)

[12] V. Vennin, H.Assadullahi, H. Firouzjahi, M.Noorbala and D. Wands, Phys.Rev.Lett.118,031301(2017)

[13] H.Assadullahi, H. Firouzjahi, M.Noorbala,V. Vennin and D. Wands, JCAP06(2016)043

[14] N. Ikeda and S. Watanabe, Stochastic Differential Equations and Diffusion Processes, North Holland,1981

[15] T. Josset, A.Perez and D. Sudarsky, Phys.Rev.Lett.118,021102(2017)

[16] A. Berera and Li-Zhi Fang, Phys.Rev.Lett.74,1912(1995)

[17] A. Berera,I.G. Moss and R.O.Ramos, Rep.Progr.Phys.72,026901(2009)

[18] M.H. Hall, I.G. Moss and A.Berera, Phys.Rev.D69,083525(2004)

[19] R.O. Ramos and L.A. da Silva, JCAP03(2013)032

[20] V. Vennin and AA. Starobinsky, Eur.Phys.J. C75,413(2015)

[21] M. Liguori, S. Matarrese, M.A. Musso and A. Riotto, JCAP08(2004)011

[22] Z. Haba, Eur.Phys.J.C78,596(2018)

[23] Z. Haba, Acta Physica Polonica, Proceed.Suppl.10,333(2017)

[24] Z. Haba, Adv.High Energy Phys.2018204952(2018)

[25] A. Berera, Phys.Rev.D54,2519(1996)

[26] A.A. Starobinsky and J. Yokoyama, Phys.Rev.D50,6357(1994)

[27] J.M. Overduin and F.I. Cooperstock, Phys.Rev.D58,043506(1998) 
[28] Wei Chen and Yong-Shi Wu, Phys. Rev.D41,695(1990)

[29] E.L.D. Perico,J.A.S. Lima, S. Basilakos and J. Sola, Phys.Rev.D88,063531(2013)

[30] M. Szydlowski and A. Stachowski, JCAP10(2015)066

[31] P. Steinhardt and M.S. Turner, Phys. Rev.D29,2162(1984)

[32] W. Lee and Li-Zhi Fang, Phys.Rev.D59,083503(1999)

[33] I.I. Gikhman and A.B. Skorohod, Stochastic Differential Equations, Springer, 1972

[34] D.S. Salopek and J.R. Bond, Phys.Rev.42,3936(1990)

[35] A.R. Liddle, P. Parsons and J.D. Barrow, Phys.Rev.D507222(1994)

[36] J.E. Lidsey, A.R. Liddle, E.W. Kolb, E.J. Copeland, T. Barreiro and M. Abney,Rev. Mod.Phys.69,374(1997)

[37] A.D. Rendall, Class.Quant.Grav.22,1655(2005)

[38] A.A. Starobinsky, JETP Lett.42,152(1985)

[39] H. Risken, The Fokker-Planck Equation, Berlin,1989

[40] J. Martin, C. Ringeval and V.Vennin, Encyclopedia Inflationaris, arXiv: 1303.3787

[41] K. Bamba, S. Nojiri and S.D. Odintsov, Phys.Lett.B737,374(2014)

[42] P.J.E. Peebles and B. Ratra, Astroph.J.325,L17(1988)

[43] P.J.E. Peebles and A. Vilenkin, Phys.Rev.D60,103506(1999)

[44] A. Linde, Phys.Lett.B129,177(1983)

[45] K. Freese, J.A. Frieman and A.V. Olinto, Phys.Rev. Lett.65,3233(1990)

[46] J.-P. Eckmann and D. Ruelle, Rev.Mod.Phys.57,617(1985)

[47] R.J. Hardwick, V.Vennin, Ch.T. Byrnes, J. Torrado and D. Wands, arXiv:1701.06473

[48] T. Fujita, M.Kawasaki,Y.Tada and T. Takesako, JCAP12(2013)036

T. Fujita, M. Kawasaki and Y. Tada, JCAP10(2014)030 